\title{
Implementation of neuro-oncology service reconfiguration in accordance with NICE guidance provides enhanced clinical care for patients with glioblastoma multiforme
}

\author{
MR Guilfoyle', RA Weerakkody', A Oswal', I Oberg', C Jeffery', K Haynes', PJ Kullar', D Greenberg², \\ SJ Jefferies ${ }^{3}$, F Harris ${ }^{3}$, SJ Price', S Thomson ${ }^{*, 4,5}$ and C Watts ${ }^{*, 1,5}$ \\ 'Cambridge University Department of Clinical Neurosciences, Addenbrooke's Hospital, Hills Road, Cambridge CB2 OQQ, UK; ${ }^{2}$ Eastern Cancer \\ Registration and Information Centre, Magog Court, Shelford Bottom, Cambridge CB22 3AD, UK; ${ }^{3}$ Department of Oncology, Addenbrooke's Hospital, Hills \\ Road, Cambridge CB2 OQQ, UK; ${ }^{4}$ Department of Neurosurgery, Leeds General Infirmary, Leeds LSI 3EX, UK
}

BACKGROUND: Brain tumours account for $<2 \%$ of all primary neoplasms but are responsible for $7 \%$ of the years of life lost from cancer before age 70 years. The latest survival trends for patients with CNS malignancies have remained largely static. The objective of this study was to evaluate the change in practice as a result of implementing the Improving Outcomes Guidance from the UK National Institute for Health and Clinical Excellence (NICE).

METHODS: Patients were identified from the local cancer registry and hospital databases. We compared time from diagnosis to treatment, proportion of patients discussed at multidisciplinary team (MDT) meetings, treatment received, length of inpatient stay and survival. Inpatient and imaging costs were also estimated.

RESULTS: Service reconfiguration and implementation of NICE guidance resulted in significantly more patients being discussed by the MDT-increased from 66 to $87 \%$, reduced emergency admission in favour of elective surgery, reduced median hospital stay from 8 to 4.5 days, increased use of post-operative MRI from 17 to $91 \%$ facilitating early discharge and treatment planning, and reduced cost of inpatient stay from $£ 2096$ in 2006 to $£ 1316$ in 2009. Patients treated with optimal surgery followed by radiotherapy with concomitant and adjuvant temozolomide achieved outcomes comparable to those reported in clinical trials: median overall survival 18 months (2-year survival 35\%).

CONCLUSIONS: Advancing the management of neuro-oncology patients by moving from an emergency-based system of patient referral and management to a more planned elective outpatient-based pattern of care improves patient experience and has the potential to deliver better outcomes and research opportunities.

British Journal of Cancer (2011) 104, I810-1815. doi:10.1038/bjc.2011.153 www.bjcancer.com

Published online 24 May 2011

(C) 2011 Cancer Research UK

Keywords: glioblastoma; management; surgery; radiotherapy; chemotherapy; NICE guidance

Each year in the UK over 4000 new cases of brain or central nervous system cancers are diagnosed, $\sim 7$ per 100000 of the population (Rachet et al, 2008). Although brain tumours account for $<2 \%$ of all primary neoplasms high mortality rates mean they rank as the third leading cause of cancer-related death among men aged 15-54 years and the fourth leading cause of cancer-related death among women between 15 and 34 years of age (Kesari and Stiles, 2006). Considered in terms of years of life lost brain tumours are responsible for the highest cancer burden, with an average of over 20 years lost per patient (Burnet et al, 2005).

The commonest primary brain tumours in adults are astrocytomas, of which the majority are malignant glioblastoma (GBM). Survival trends for primary CNS malignancy have remained largely static for many years, reflecting the general lack of effective

\footnotetext{
*Correspondence: Dr C Watts; E-mail: cw209@cam.ac.uk

or S Thomson; E-mail: simon.thomson@leedsth.nhs.uk

${ }^{5}$ These authors contributed equally to this work.

Received 5 October 2010; revised 23 March 2011; accepted 7 April 201 I; published online 24 May 2011
}

therapeutic options for patients with these cancers (Rachet et al, 2008). Clinical management of patients with GBM involves a combination of surgery, radiotherapy and chemotherapy. Radiotherapy has been the principle adjuvant modality since the late 1970s (Walker et al, 1978, 1980) with the addition of chemotherapy demonstrating only modest benefit until recently (Stewart, 2002; Stupp et al, 2005). Current median life expectancy with optimal treatment is $12-14$ months, with approximately a quarter of patients surviving $>24$ months (Stupp et al, 2005). There clearly remains an unmet clinical need for new therapeutic interventions.

Improving clinical outcomes not only requires the development and application of more effective treatment but also better organisation of the delivery of care (NICE, 2006). Historically, patients with brain cancer have often experienced a fragmented and uncoordinated pathway of care from diagnosis through to treatment and follow-up. The prevailing pessimism towards the efficacy of surgery for GBM contributed to a lack of prompt treatment after diagnosis, a general policy of biopsy in preference to resection, subtotal debulking when undertaken and little 
urgency in the commencement of adjuvant therapy. Recent evidence has clearly demonstrated that substantial gains in survival are possible with new advanced surgical techniques and oncological management (Stupp et al, 2005; Stummer et al, 2006). The maximal benefits of these developments will only be realised with coordinated and specialised interdisciplinary care delivering best-available treatment for each individual patient in a timely manner. Alongside these changes in practice, health professionals have an obligation to ensure patients can make informed decisions about the complex treatment choices being presented to them. The UK National Institute of Clinical Excellence (NICE), in its Improving Outcomes Guidance (IOG) for people with brain and other CNS tumours, has identified key aspects of neuro-oncology services that need development (NICE, 2006). The principal recommendations include establishing direct referral pathways, multidisciplinary teams (MDTs) of neurosurgeons, oncologists, pathologists and radiologists to review diagnoses and determine appropriate treatment for every individual, supportive pre- and post-operative counselling of patients and enhanced opportunities for participation in clinical trials (NICE, 2006).

There is considerable evidence that centre and surgeon subspecialisation has had significant benefits on the outcomes for systemic cancers (e.g., breast and colon) (Gooiker et al, 2010; van Gijn et al, 2010). Similarly, subspecialist paediatric neurosurgeons treating high caseloads of children with posterior fossa tumours obtain better rates of gross tumour resection, a major predictor of survival, and lower concomitant morbidity (Albright et al, 2000). Developing the subspecialty of adult neurosurgical oncology is an important component of the drive to improve standards of care and patient safety, to facilitate the introduction of new technologies and to promote and direct research. Dedicated neurosurgical oncology clinics provide opportunities for patients to discuss their management and consent to treatment, and also promote enrolment in research such as tissue banking and recruitment into clinical trials. The need for improvement is highlighted by the fact that over the past decade there have been 13 open trials of novel therapies for paediatric brain tumours but only 4 open trials for adults on the National Cancer Research Network (http://www.ncrn.org.uk/) trial portfolio.

NICE guidance has significant implications for the organisation of neurosurgical services and has been variably adopted by the 34 neurosurgical units in the UK. Here, we describe how the guidance was implemented and integrated within the Anglian Cancer Network and the effects this had on clinical service delivery. To set these developments in context, we have first established outcomes for patients treated in the 10 years before the NICE guidance. Second, we consider the potential problems and cost implications of introducing an outpatient-based management strategy without increasing delays in the patient pathway by auditing the length of stay and the length of the patient journey (from diagnostic scan to oncology management) before, during and after the full implementation of the guidelines. Third, we examine the realistic patient outcomes that are now achievable with service reorganisation in combination with current optimal surgical and adjuvant strategies.

\section{METHODS}

\section{Outcome of patients treated 1997-2006}

Patients were identified from the Eastern Cancer Registration and Information Centre (ECRIC) database, which collects all confirmed and suspected diagnoses of cancer from the Anglian Cancer Network. This is the third largest cancer network in the UK with an estimated population of over 2.66 million people (http://www. ncin.org.uk). Addenbrooke's Hospital, part of the Cambridge University Hospital NHS Foundation Trust (CUHFT) is the dedicated neurosurgical centre for this network. The ECRIC database was queried for ICD-10 codes $\mathrm{C}^{*} 1^{*}$ and/or ICD-O-3 codes $94^{\star *}$ for the period 1997-2006 to obtain the study sample. Hospital pathology reports were then cross-checked to confirm histological diagnosis of GBM. Patients with presumed diagnoses on the basis of imaging but without histological confirmation were excluded. Data collected included gender, date of birth, date of diagnosis, date of death and whether radiotherapy and/or chemotherapy were received in the first 6 months after diagnosis. Surgical procedure (stereotactic biopsy vs craniotomy and debulking) was queried from the operating department database.

\section{Implementation of NICE guidelines and current treatment outcomes}

Changing the neuro-oncology service in accordance with the NICE guidance evolved as an iterative process. First, a weekly multidisciplinary meeting was convened and the core members from each subspecialty were identified and attendance registered. A dedicated neurosurgical oncology clinic was established and scheduled for the afternoon on the same day to review new and follow-up patients discussed at the MDT. The second development involved changing the pattern of referral while maintaining patient safety. All physicians referring patients with suspected brain tumours completed a proforma designed to facilitate data collection transmitted electronically to the MDT coordinator (see Supplementary Material). All cases were first discussed with the on-call neurosurgical team to ensure genuine emergencies (e.g., cerebral abscess) were managed appropriately.

To evaluate change in practice resulting from the NICE IOG, we examined the management of patients during the 6 months immediately before the report (February to July 2006) and compared findings with two 6-month periods following publication (April to November 2008 and the same period in 2009). A number of indicators in the treatment pathway relating to key recommendations in the NICE guidance were examined, specifically proportions of cases discussed at MDT before and after surgical treatment, time from referral to MDT discussion, treatment, and histopathology result, length of inpatient stay and proportion of patients managed as elective admissions as opposed to emergency transfers.

Data were retrieved from the MDT database and supplemented with case-note data. We calculated the cost of inpatient stay and imaging using a standard unit cost for a bed-day of $£ 262$ and unit cost for an MRI scan of $£ 137$, derived from the hospital business intelligence system.

Difficulties in implementing the IOG included ensuring that the clinical details forwarded to the MDT were adequate to allow a consensus decision on appropriate treatment. So that sufficient data were collected a standardised proforma with a minimum data set was developed (see Supplementary Material). To facilitate the specialisation of surgical practice, we implemented a process of clinical governance to maintain efficient and safe patient management. The introduction of routine post-operative MRI scans within $72 \mathrm{~h}$ further served as a tool for audit of surgical standards, and the images were incorporated into treatment planning by the clinical oncologists. Earlier discharge of patients within $48-72 \mathrm{~h}$ of surgery offset additional imaging costs.

\section{Outcomes of contemporary adjuvant treatment}

For comparison against the historical data, we examined survival in patients currently receiving IOG compliant maximal surgical and adjuvant therapy, that is, debulking of the tumour where possible followed by concomitant radiotherapy and temozolomide chemotherapy (RT-TMZ) and subsequent further adjuvant temozolomide.

Statistical analysis was performed in SPSS 18.0 (IBM, Middlesex, UK). Survival was estimated with the Kaplan-Meier method and 
compared between groups with the log-rank test. Cox-regression analysis was used to identify predictors of survival. Continuous variables were compared with the Mann-Whitney $U$-test and dichotomous variables compared with the $\chi^{2}$-test. Results were considered significant at the $5 \%$ level.

\section{RESULTS}

\section{Improving outcomes before NICE guidance}

From 1997 to 2006, a total of 685 patients were diagnosed with histologically proven GBM. Mean age was 60.7 years (s.d. 11.4, median 62) and a majority of patients were male $(61.0 \%)$. Overall median survival for all patients was 5.03 months $(95 \% \mathrm{CI}$ 4.56-5.50; Table 1, Figure 1A). Univariate analyses (Table 1) showed significantly longer survival for patients receiving debulking surgery over stereotactic biopsy (median $6.6 \mathrm{vs} 3.8$ months, $P<0.001$; Figure 1C), radiotherapy (median 7.8 vs 2.0, $P<0.001$; Figure 1D), and sequential radiotherapy and chemotherapy, typically a procarbazine, lomustine (CCNU), and vincristine (PCV) regimen (median 14.5 vs 4.2 months, $P<0.001$; Figure 1D). As expected there was an inverse association between survival and age (median <45 years: 14.1 months, 45 - 54 years: 8.3 months, 55-64 years: 5.6 months, 65-74 years: 3.7 months, $>75$ years: 2.8 months, $P<0.001$; Figure $1 \mathrm{~B})$.

The first (1997-2001) and latter (2002-2006) halves of the study period were compared to assess changes in practice and outcome (Figure 2). There was no change in patient age (mean $61.5 \mathrm{vs}$ 59.8 years, $t=1.95, P>0.05$ ) or gender (male: $58.9 \%$ vs $63.7 \%$, $\left.\chi^{2}=1.7, P>0.05\right)$. However, there were significant increases in the proportions of patients receiving debulking surgery $(39.9 \%$ vs $54.8 \%$, $\left.\chi^{2}=13.8, P<0.001\right)$, adjuvant radiotherapy $\left(59.4 \%\right.$ vs $66.8 \%, \chi^{2}=3.9$, $P=0.048)$ and chemotherapy $\left(10.4 \%\right.$ vs $28.0 \%, \chi^{2}=20.2, P<0.001$; Figure $2 \mathrm{~A}$ ). There was a significant improvement in survival between the two 5-year periods (4.4 vs 5.8 months, $P<0.001$; Figure $2 \mathrm{~B}$ ). Coxregression analysis showed the independent predictors of survival were age (Hazard ratio (95\%CI): $1.03(1.02-1.04), P<0.001)$, debulking surgery (HR $0.80(0.67-0.94), P<0.01)$, radiotherapy (HR $0.43(0.36-0.51), P<0.001)$ and chemotherapy (HR 0.59
(0.46-0.75), $P<0.01)$. Gender and year of diagnosis were not significantly associated with survival in the model.

\section{Implementation of NICE guidelines}

Over a period of $\sim 10$ months, the surgical management of neurooncology patients was gradually transferred to three of the ten consultant neurosurgeons and the neurosurgical oncology clinic was firmly established. Neuro-oncology was formally defined as a
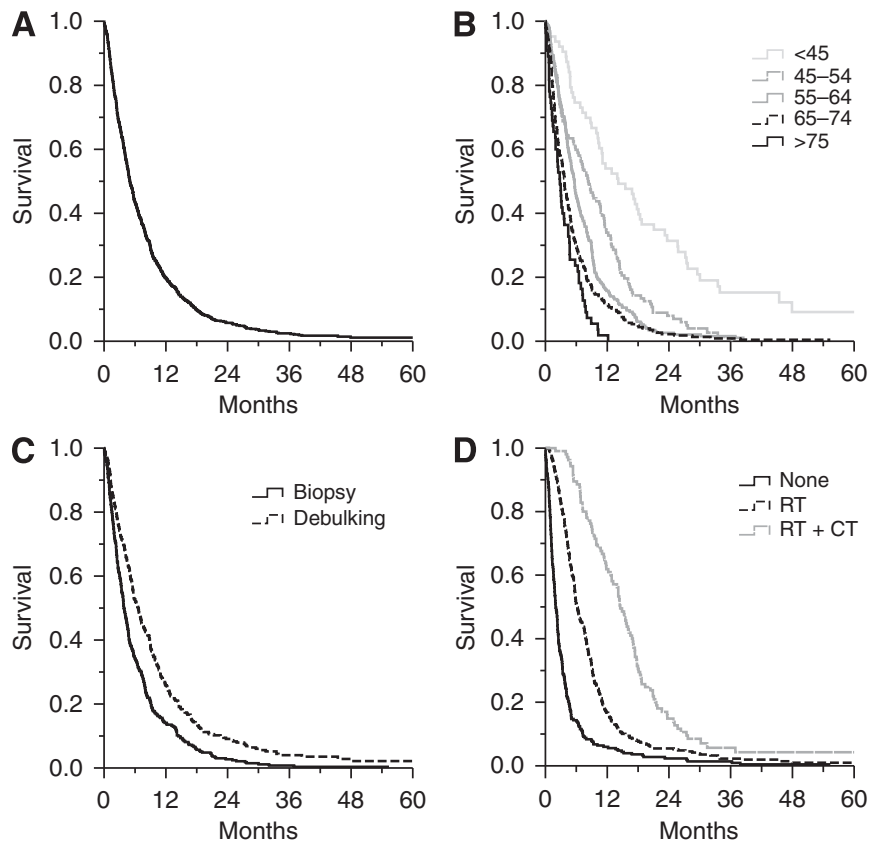

Figure I Survival for historical cohort. Kaplan-Meier curves for patients with GBM diagnosed 1997-2006. Survival of all patients (A), stratified by age (B), comparing stereotactic biopsy and tumour debulking $(\mathbf{C})$ and comparing sequential radiotherapy and chemotherapy (D).

Table I Historical survival of GBM patients

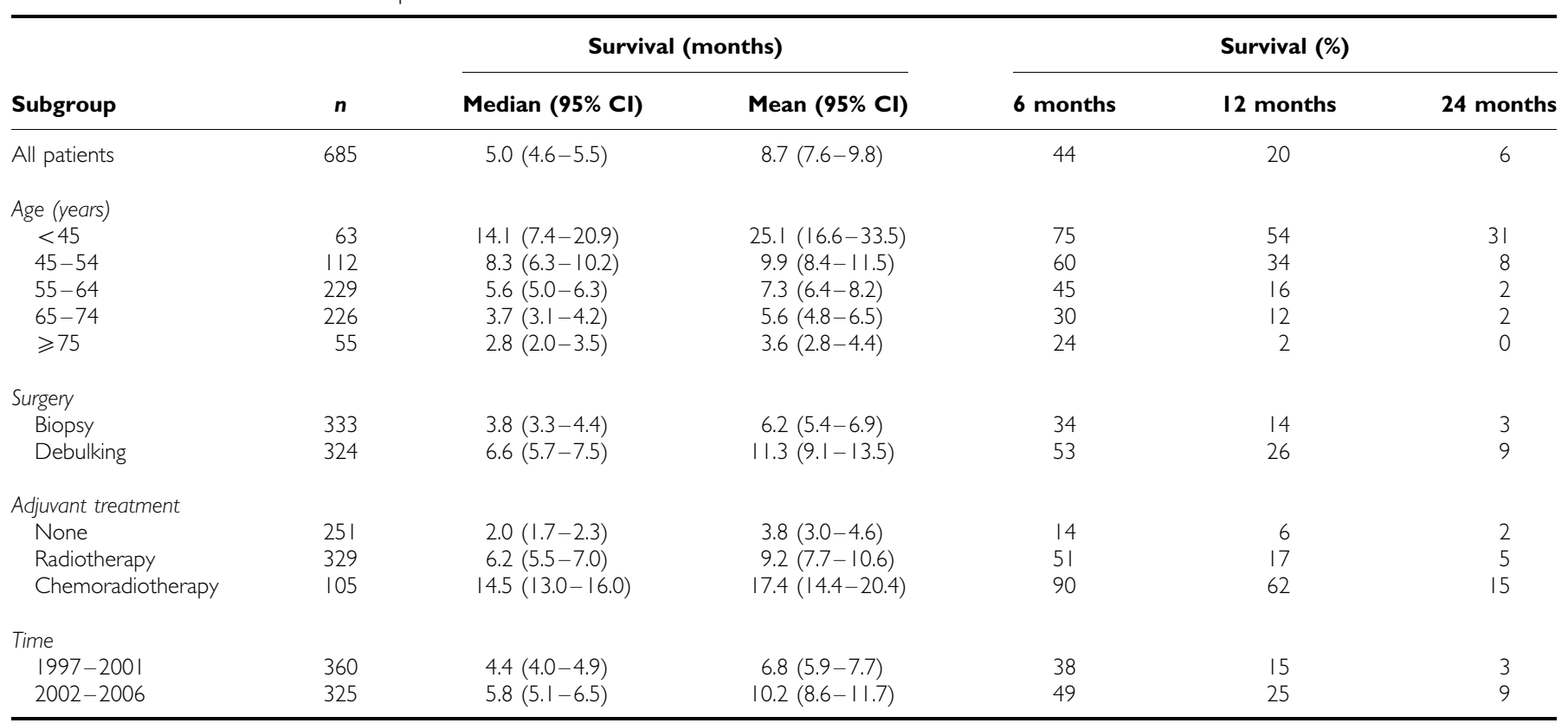

Abbreviations: $\mathrm{Cl}=$ confidence interval; $\mathrm{GBM}=$ glioblastoma. 
BJC $\cap$
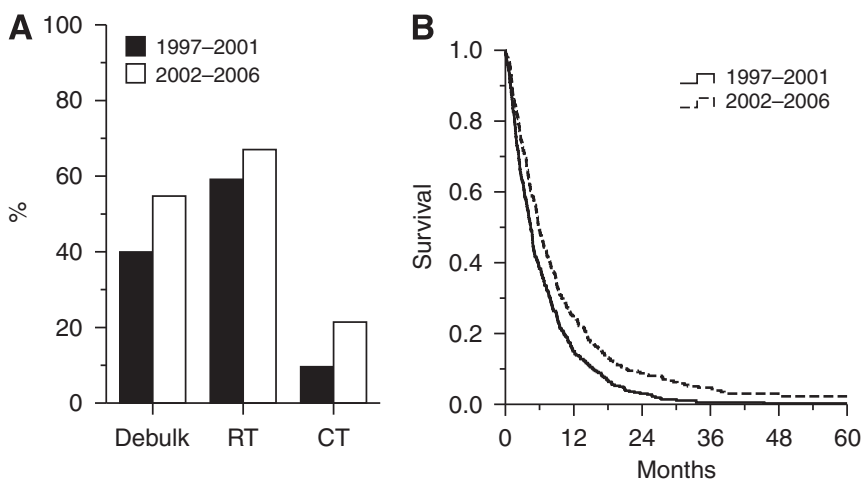

Figure 2 Change in management and outcome. Proportions of patients receiving tumour debulking surgery (vs biopsy) and adjuvant radiotherapy (RT) and chemotherapy (CT) in the two 5-year periods of the study $\left(\right.$ (A) all comparisons with $\chi^{2}$-test, $\left.P<0.05\right)$. Kaplan-Meier curves for the two study periods (B). Comparison with log-rank test shows significant improvement in survival $(P<0.00 \mathrm{I})$

neurosurgical subspecialty, eventually leading to the appointment of a clinical lead for neuro-oncological surgery in September 2007 and an additional neurosurgeon with a subspecialty interest in neuro-oncology was appointed in April 2008. The surgeons specialising in neuro-oncology easily fulfilled the IOG requirement for $>50 \%$ of a surgeon's practice to comprise tumour work.

Thirty-five patients were diagnosed with GBM during period 1 (February to July 2006) increasing to 49 and 45 in periods 2 (April to November 2008) and 3 (April to November 2009), respectively (total $n=129)$. The proportion of cases reviewed at an MDT meeting before surgery significantly increased (66-87\%, $P=0.027$; Figure $3 \mathrm{~A})$. The proportion of patients undergoing MRI within $72 \mathrm{~h}$ of tumour debulking increased from 17 to $91 \%(P<0.001$; Figure $3 \mathrm{~A}$ ). In period 1 , most patients were admitted for surgery as emergencies, whereas in both periods 2 and 3 the majority were admitted electively as urgent (not emergency) cases $(P<0.001$; Figure $3 \mathrm{~B}$ ). Median length of stay was 8 days in period 1, decreasing to 4.5 days in period 3 ( $P=0.004$; Figure $3 C)$. Median time for histopathology reporting decreased from 6 to 3 days $(P<0.001)$; all cases and results were reviewed at a post-operative MDT meeting. Overall time from referral imaging to post-operative MDT remained unchanged (median 23 days; $P=0.61$ ). The proportion of patients receiving their diagnosis in a specialist neuro-oncology clinic increased from 65 to $100 \%(P<0.001)$ and the time from operation to outpatient review significantly decreased (median 17 vs 10 days; $P<0.001)$. At the same time, we observed a significant trend in reducing total cost of inpatient stay and imaging (median costs 2006: $£ 2096,2008$ : $£ 1048,2009$ : $£ 1316, P<0.01$; Figure 3D).

\section{Outcomes of contemporary optimal treatment}

The implementation of RT with concomitant and adjuvant TMZ occurred in April 2005 following specific CUHFT approval and funding on an individual patient basis, predating the NICE technology appraisal by 2 years (NICE, 2007). From 2005 to 2008, 49 patients aged 26-68 years (mean 56) were commenced on the RT-TMZ protocol. Consistent with published data, 92\% completed the concomitant phase and $42 \%$ completed the adjuvant phase of treatment. Patients not receiving TMZ were managed with sequential radiotherapy and PCV chemotherapy according to standard best practice. Outcomes were similar to the 2002-2006 cohort and the data were not analysed further.

Patients for RT-TMZ were selected as per the original Stupp trial protocol (Stupp et al, 2005). All were WHO performance status 0 or 1 with mean age 56 years, and $80 \%$ had undergone debulking surgery. Of those starting combined therapy 43 (88\%) completed
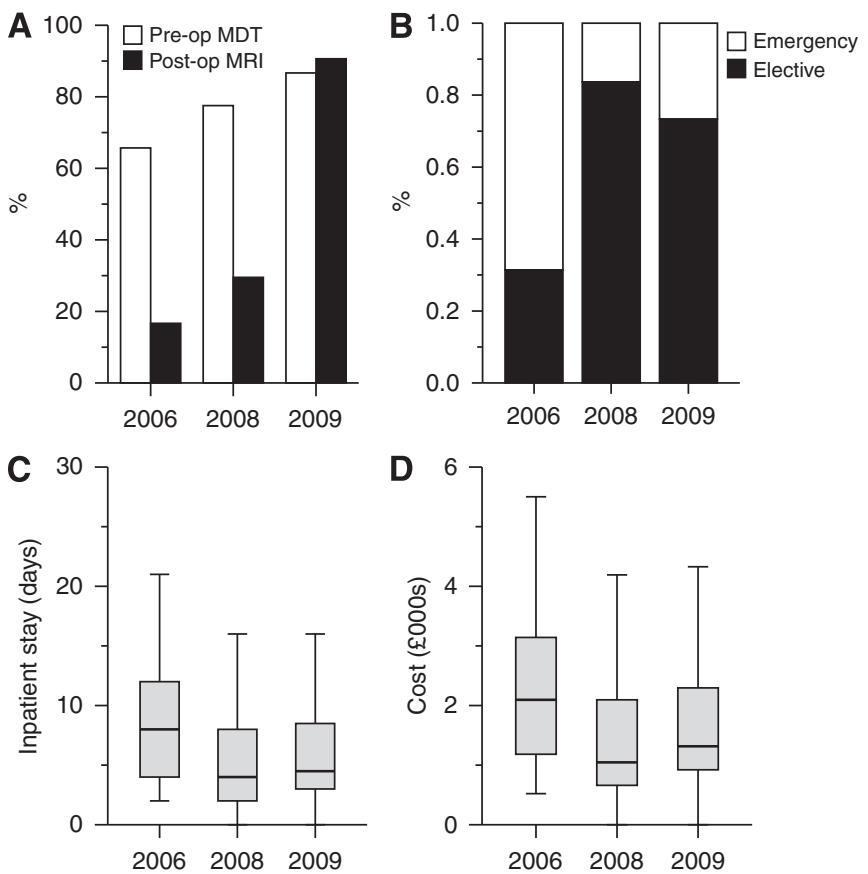

Figure 3 Implementation of NICE guidelines. Proportions of cases discussed at pre-operative MDT for management planning and receiving post-operative $\mathrm{MRI}(\mathbf{A})$. Change in proportion of patients admitted as emergencies or as urgent elective cases (B). Box and whisker plots of length of stay $(\mathbf{C})$ and cost of inpatient stay plus imaging (D). Box represents interquartile range with median marked by solid bar.
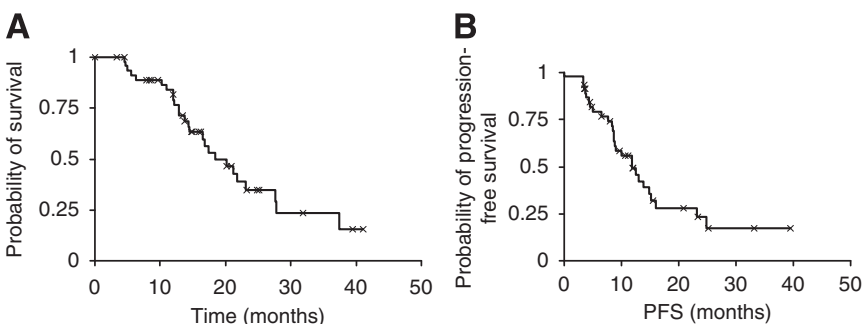

Figure 4 Survival with modern optimal RT-TMZ treatment. KaplanMeier curves for overall survival (median OS, 18 months $(\mathbf{A})$ ) and clinical progression-free survival (PFS, 12 months $(\mathbf{B})$ ) of patients treated with concomitant and adjuvant temozolomide. Two-year survival is 35\%.

the concomitant TMZ; treatment was discontinued in three patients due to abnormal liver function tests, two due to thrombocytopaenia and one was unable to tolerate TMZ because of nausea. Twenty-four patients $(53 \%)$ did not complete the adjuvant phase of TMZ treatment due to evidence of radiological progression in 9 and clinical progression in 10, deranged liver enzymes in 2 patients, significant Herpes Zoster infection in 2 and intolerance in 1. Median overall survival (OS) and clinical progression-free survival (PFS) were 18 and 12 months, respectively, with 2-year survival of 35\% (Figure 4).

\section{DISCUSSION}

We present data showing how implementation of NICE IOG for service organisation together with advances in management has delivered improved standards of care and overall outcome for patients with GBM. Before the introduction of the IOG in 2006, there was a modest improvement in patient survival in the period 2002-2006 compared with 1997-2001. This was associated with 
increased access to surgical debulking, radiotherapy and chemotherapy, which were also independent predictors of survival, consistent with earlier observational studies and randomised trials (Curran et al, 1993; Brenner and Rachet, 2004; Stupp et al, 2005; Pichlmeier et al, 2008; Rachet et al, 2008).

Publication of the NICE guidance on improving the process of care for patients with CNS malignancies coincided with the advent of new evidence-based surgical techniques and oncological therapies in the first half of the last decade. These developments provided the impetus to re-evaluate and restructure brain tumour service provision for the Anglian Cancer Network. The existing patient pathway of care was mapped to define the pattern of referrals to the neurosurgical service and to identify how this could be changed to facilitate referrals to a single specialised neurosurgical clinic in line with NICE guidance. The neuro-oncology MDT was designated as the primary route of referral for all new brain tumour diagnoses and appropriate data collection implemented.

The development of a surgeon-led subspecialist clinic involving clinical nurse practitioners (CNPs) following each MDT meeting facilitated rapid review of patients to discuss diagnosis and management. Three dedicated oncology neurosurgeons and two neuro-oncology CNPs support the clinic enabling it to run uninterrupted every week. This has been fundamental in the transition from a system predominantly of inpatient transfers from district hospitals, to an outpatient-based service.

At a patient's initial clinic visit sufficient time is allotted for introduction to the team members, for in-depth discussion of provisional diagnosis and recommended management, and to begin the process of consent using procedure-specific consent forms and clear written material provided before admission for surgery (see Supplementary Material). Histological reports are generally discussed at the MDT the week after surgery and patients attend the clinic the same day to be informed of their diagnosis. At the follow-up post-operative clinic appointments difficult information, chiefly histopathology results and prognosis, are communicated in a familiar environment by clinicians the patient has met previously. There is also opportunity for wound assessment, removal of clips or sutures as necessary, weaning of steroids and review of anti-convulsants. Contact details of the CNPs are provided for ongoing support at home and to address any questions. Logistically, the clinic facilitates planning of operating lists well in advance, better coordination of preoperative imaging, day-of-surgery admissions and overall more efficient utilisation of theatre time by minimising cancellations.
Discharge planning can also start before admission with the focus on returning home rather than to local hospitals. Safe but early discharge has become the objective of occupational and physiotherapists, and the expectation of patients. The measures put in place have contributed to a streamlined service in which patients remain at home for longer, obtain treatment that is based on up to date evidence, and are able to discuss their condition in the privacy of a clinic.

The benefits of enhancing the role of pre- and post-operative MDT and restructuring the system as an outpatient-based service may potentially come at the expense of increasing delays in management, for example, from diagnostic scan to treatment. In practice, the present data demonstrate that length of stay and time from surgery to definitive pathology reporting have both been significantly reduced, and the proportion of patients having postoperative MRI within $72 \mathrm{~h}$ has increased. Furthermore, in conjunction with the shift from an emergency to an elective-based process of patient admission these measures have resulted in a net cost reduction per patient. Previous studies have suggested that neurosurgical oncology subspecialisation is of limited benefit (Latif et al, 1998), but this observation is at odds with evidence from other clinical oncology disciplines (Gooiker et al, 2010; van Gijn et al, 2010). Our data generate the testable hypothesis that an integrated specialist process of care will improve the patient experience and provide a platform to gather this data.

The litmus test of any service restructuring will be improved survival of patients in the long term. Our early experience with combining aggressive surgical resection and adjuvant chemoradiotherapy with temozolomide has been very favourable. We found a median OS, median PFS and 2-year survival of 18 months, 12 months and $35 \%$, respectively. This is comparable to the Stupp trial which reported an OS of 14.6 months, a PFS of 7 months and a 2-year survival of $26.5 \%$ (Stupp et al, 2005). Of note, our data more closely mirror the subgroup of trial patients in whom a complete surgical resection was obtained whose OS was 18.8 months and 2-year survival of $38.4 \%$ (Stupp et al, 2009). The acquisition of early post-operative baseline MRI allowed better recognition and differentiation of tumour progression and pseudoprogression so that patients did not have their adjuvant temozolomide terminated prematurely (de Wit et al, 2004; Chamberlain et al, 2007). These data represent a highly selected cohort from a single cancer network and cannot be extrapolated to a broader population. Nevertheless, they show that the service is able to deliver outcomes comparable to those achieved within a clinical trial setting.

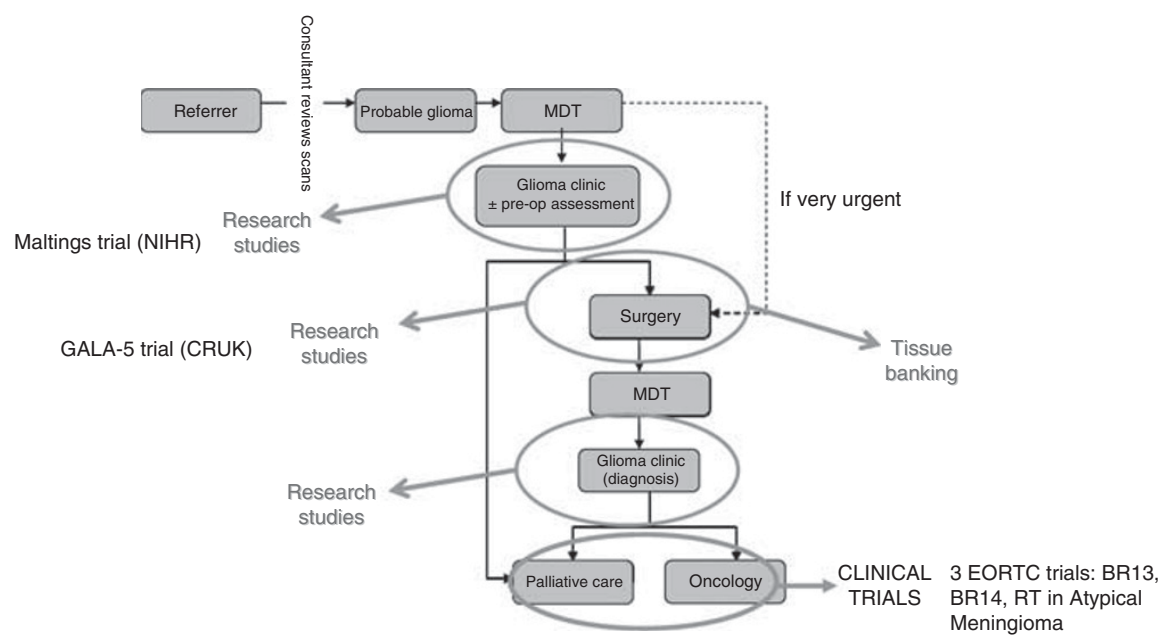

Figure 5 Service reconfiguration supports research for patient benefit. Our ability to interrogate each stage of the patient journey with an array of screening, diagnostic and analytical tools is unique. This results in a highly integrated research infrastructure capable of delivering tangible results evidenced by the number of clinical studies into which we are recruiting patients. NB: RT in Meningioma, MALTINGS and GALA-5 are all led by Cambridge Pls. 
More recently, fluorescence-guided surgical resection has been introduced and an increasing proportion of patients are receiving carmustine chemotherapy wafer implants after debulking (Westphal et al, 2003; Stummer et al, 2006). The improvements made in the system of care will ensure that all patients eligible for these advanced interventions are offered current best treatment. The introduction of a dedicated referral pathway, specialist MDT meetings and routine postoperative imaging has established a robust mechanism for rigorous surgical, radiological and chemotherapy audit and provides the necessary platform for continued research and recruitment into clinical trials (Figure 5). The latter benefit is evidenced by the opening of a NIHR-funded observational study imaging tumour invasion (MALTING Study; UKCRN 8596) and a CRUK/SDBTT funded phase 1 study combining intraoperative chemotherapy with fluorescenceguided resection (GALA-5; CRUK/10/009). Improved access to tissue has enabled us to refine a protocol for efficient derivation of stem-like tumour initiating cells from GBM and to develop pre-clinical animal models for drug development and testing (Fael Al-Mayhani et al, 2009).

We show that neurosurgical oncology services can be reconfigured from an unplanned, consultant centric, research ambivalent process of care into an outpatient-based, consultant led, patientcentred, research-orientated practice. We have established that these changes are cost effective. Our experience suggests that wider implementation of NICE IOG-based models of service delivery will benefit patients, clinicians, researchers and primary care commissioners.

\section{ACKNOWLEDGEMENTS}

Colin Watts is a HEFCE Clinical Senior Lecturer. Stephen Price is a NIHR Clinician Scientist. Mathew Guilfoyle is supported by the Philip King/Royal College of Surgeons of England Research Fellowship. We would like to thank the William Brown Trust for their support, and all the staff at ECRIC for their help in the preparation of this manuscript.

Supplementary Information accompanies the paper on British Journal of Cancer website (http://www.nature.com/bjc)

\section{REFERENCES}

Albright AL, Sposto R, Holmes E, Zeltzer PM, Finlay JL, Wisoff JH, Berger MS, Packer RJ, Pollack IF (2000) Correlation of neurosurgical subspecialization with outcomes in children with malignant brain tumors. Neurosurgery 47(4): $879-887$

Brenner H, Rachet B (2004) Hybrid analysis for up-to-date long-term survival rates in cancer registries with delayed recording of incident cases. Eur J Cancer 40(16): 2494-2501

Burnet NG, Jefferies SJ, Benson RJ, Hunt DP, Treasure FP (2005) Years of life lost (YLL) from cancer is an important measure of population burden and should be considered when allocating research funds. $\mathrm{Br}$ Cancer 92(2): $241-245$

Chamberlain MC, Glantz MJ, Chalmers L, Van Horn A, Sloan AE (2007) Early necrosis following concurrent Temodar and radiotherapy in patients with glioblastoma. J Neurooncol 82(1): $81-83$

Curran Jr WJ, Scott CB, Horton J, Nelson JS, Weinstein AS, Fischbach AJ, Chang CH, Rotman M, Asbell SO, Krisch RE, Nelson DF (1993) Recursive partitioning analysis of prognostic factors in three radiation therapy oncology group malignant glioma trials. J Natl Cancer Inst 85(9): 704 - 710

de Wit MC, de Bruin HG, Eijkenboom W, Sillevis Smitt PA, van den Bent MJ (2004) Immediate post-radiotherapy changes in malignant glioma can mimic tumor progression. Neurology 63(3): 535-537

Fael Al-Mayhani TM, Ball SL, Zhao JW, Fawcett J, Ichimura K, Collins PV, Watts C (2009) An efficient method for derivation and propagation of glioblastoma cell lines that conserves the molecular profile of their original tumours. J Neurosci Methods 176(2): 192-199

Gooiker GA, van Gijn W, Post PN, van de Velde CJ, Tollenaar RA, Wouters MW (2010) A systematic review and meta-analysis of the volumeoutcome relationship in the surgical treatment of breast cancer. Are breast cancer patients better of with a high volume provider? Eur J Surg Oncol 36(Suppl 1): S27-S35

Kesari S, Stiles CD (2006) The bad seed: PDGF receptors link adult neural progenitors to glioma stem cells. Neuron 51(2): 151-153

Latif AZ, Signorini DF, Whittle IR (1998) Treatment by a specialist surgical neuro-oncologist does not provide any survival advantage for patients with a malignant glioma. Br J Neurosurg 12(1): 29-32

NICE (2006) Service guidance for improving outcomes for people with brain and other central nervous system tumours. Retrieved 1 January 2011, http://www.nice.org.uk/nicemedia/pdf/CSG_brain_manual.pdf

NICE (2007) TA121 glioma (newly diagnosed and high grade) - carmustine implants and temozolomide: guidance. Retrieved 1 January 2011, http:// www.nice.org.uk/nicemedia/pdf/TA121guidance.pdf

Pichlmeier U, Bink A, Schackert G, Stummer W, ALA Glioma Study Group (2008) Resection and survival in glioblastoma multiforme: an RTOG recursive partitioning analysis of ALA study patients. Neuro Oncol 10(6): $1025-1034$

Rachet B, Mitry E, Quinn MJ, Cooper N, Coleman MP (2008) Survival from brain tumours in England and Wales up to 2001. Br J Cancer 99(S1): S98 - S101
Stewart L (2002) Chemotherapy in adult high-grade glioma: a systematic review and meta-analysis of individual patient data from 12 randomised trials. Lancet 359(9311): 1011-1018

Stummer W, Pichlmeier U, Meinel T, Wiestler OD, Zanella F, Reulen HJ (2006) Fluorescence-guided surgery with 5-aminolevulinic acid for resection of malignant glioma: a randomised controlled multicentre phase III trial. Lancet Oncol 7(5): 392-401

Stupp R, Hegi ME, Mason WP, van den Bent MJ, Taphoorn MJ, Janzer RC, Ludwin SK, Allgeier A, Fisher B, Belanger K, Hau P, Brandes AA, Gijtenbeek J, Marosi C, Vecht CJ, Mokhtari K, Wesseling P, Villa S, Eisenhauer E, Gorlia T, Weller M, Lacombe D, Cairncross JG, Mirimanoff RO (2009) Effects of radiotherapy with concomitant and adjuvant temozolomide versus radiotherapy alone on survival in glioblastoma in a randomised phase III study: 5-year analysis of the EORTC-NCIC trial. Lancet Oncol 10(5): 459-466

Stupp R, Mason WP, van den Bent MJ, Weller M, Fisher B, Taphoorn MJ, Belanger K, Brandes AA, Marosi C, Bogdahn U, Curschmann J, Janzer RC, Ludwin SK, Gorlia T, Allgeier A, Lacombe D, Cairncross JG, Eisenhauer E, Mirimanoff RO, European Organisation for Research and Treatment of Cancer Brain Tumor and Radiotherapy Groups; National Cancer Institute of Canada Clinical Trials Group (2005) Radiotherapy plus concomitant and adjuvant temozolomide for glioblastoma. N Engl J Med 352(10): 987-996

van Gijn W, Gooiker GA, Wouters MW, Post PN, Tollenaar RA, van de Velde CJ (2010) Volume and outcome in colorectal cancer surgery. Eur J Surg Oncol 36(Suppl 1): S55-S63

Walker MD, Alexander Jr E, Hunt WE, MacCarty CS, Mahaley Jr MS, Mealey Jr J, Norrell HA, Owens G, Ransohoff J, Wilson CB, Gehan EA, Strike TA (1978) Evaluation of BCNU and/or radiotherapy in the treatment of anaplastic gliomas. J Neurosurg 49(3): $333-343$

Walker MD, Green SB, Byar DP, Alexander Jr E, Batzdorf U, Brooks WH, Hunt WE, MacCarty CS, Mahaley Jr MS, Mealey Jr J, Owens G, Ransohoff 2nd J, Robertson JT, Shapiro WR, Smith Jr KR, Wilson CB, Strike TA (1980) Randomized comparisons of radiotherapy and nitrosoureas for the treatment of malignant glioma after surgery. $N$ Engl J Med 303(23): $1323-1329$

Westphal M, Hilt DC, Bortey E, Delavault P, Olivares R, Warnke PC, Whittle IR, Jääskeläinen J, Ram Z (2003) A phase 3 trial of local chemotherapy with biodegradable carmustine (BCNU) wafers (Gliadel wafers) in patients with primary malignant glioma. Neuro Oncol 5(2): 79-88

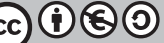

This work is licensed under the Creative Commons Attribution-NonCommercial-Share Alike 3.0 Unported License. To view a copy of this license, visit http://creativecommons. org/licenses/by-nc-sa/3.0/ 